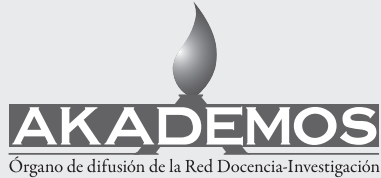

ISSN: 1995-4743

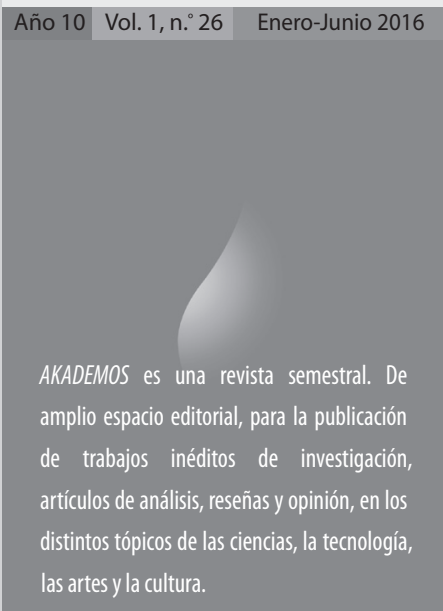

San Salvador, El Salvador, Centroamérica

\title{
La importancia de la gestión de proyectos en la industria de la construcción
}

Francisco Sorto Rivas

Docente de la Facultad de Posgrados y Educación Continua, Máster en Economía*

fran.sorto@gmail.com

\section{Resumen}

En este artículo se resalta la importancia que tiene la gestión profesional de proyectos de construcción, para el éxito financiero de los contratistas, ya que muchas veces se descuida la labor de seguimiento de las obras, ignorándose a veces inclusive el cronograma de entregables, sujeto a las restricciones presupuestarias delimitadas durante la etapa de planificación.

El control oportuno sirve para identificar desviaciones en las fechas de entrega programadas y en relación con el presupuesto original; a partir de ello, se pueden implementar medidas correctivas que eviten demoras inaceptables para los contratantes y que impidan la elevación de los costos más allá de la conveniencia financiera de los contratistas.

El descuido en el control de los proyectos puede provocar, incluso, el abandono de proyectos o la ruina de los empresarios de la construcción.

En todo caso, este control tiene por finalidad tomar decisiones oportunas para recuperar el tiempo perdido y evitar la sangría financie-

Agradezco a la señora Cristina de Chávez por su apoyo para la redacción de este artículo. 
ra detectada, en un momento determinado, durante la ejecución de los proyectos.

Para garantizar una gestión responsable y profesional de los proyectos de construcción existen guías elaboradas por el Project Managment Institute, Inc. y que forman parte del diseño curricular de los programas de posgrado relacionados con la gestión de proyectos, tal como sucede con la Universidad Doctor José Matías Delgado.

Palabras Clave: Análisis del Valor Ganado; Curvas S; Estructura de Desglose de Trabajo; Fecha Estado; Gestión de Proyectos; Índices de Desempeño; Valor Ganado.

\section{Desarrollo:}

\section{Introducción}

La evaluación de proyectos es una precondición para la asignación eficiente de los recursos productivos de una organización, ya que antes de adquirir activos con los cuales las empresas obtendrían ingresos futuros, resulta necesario determinar su costo a efecto de cerciorarse que los ingresos futuros esperados de la ejecución del proyecto, expresados a valor presente, superarían el costo de oportunidad de dichos recursos, en caso que éstos se hubieran invertido en otro emprendimiento con un nivel de riesgo equivalente.

Esta evaluación puede verse afectada, posteriormente, al implementarse el proyecto, a consecuencia de un ineficiente control de ejecución.
Esto último resulta crítico para los proyectos de construcción, dado que la programación de las actividades debe ceñirse a parámetros de tiempos de entrega de las obras, especificaciones técnicas y niveles aceptables de tolerancia en costos; esta planificación puede verse afectada por eventos ajenos al control de los contratistas, inclusive cuando se hayan realizado los estudios de suelo apropiados; pero aspectos del clima, programación de suministros y la gestión del recurso humano constituyen factores de riesgo importantes que pueden elevar, significativamente, los costos o extender los plazos de entrega, más allá de márgenes de ganancia aceptables para los inversionistas.

Claro está que existen riesgos que se pueden gestionar con facilidad al contratar seguros, disponer de garantías o acceso a algún tipo de fianza, trasladando con ello hacia terceros eventuales pérdidas derivadas de eventos fortuitos que puedan perjudicar el desarrollo normal de las obras.

\section{Gestión de proyectos de construcción}

Los proyectos de construcción requieren, por lo tanto, de una planificación detallada de actividades y de su agrupación en "paquetes de trabajo" que permitan medir, objetivamente, el avance de las obras, así como su organización de manera secuencial, ciñéndose a la ruta crítica elaborada para tales efectos.

A este agrupamiento de tareas se le conoce como Estructura de Desglose de Trabajo (EDT) en el argot de la industria de la cons- 
trucción y el control riguroso de los hitos alcanzados facilita el control de tiempos, costos y la administración dinámica de los recursos físicos y humanos disponibles para la ejecución del proyecto.

La gestión de un proyecto de construcción abarca, por ende, la etapa de planificación, ejecución y control, como mínimo, para cerciorarse que los constructores obtendrán resultados financieros satisfactorios a sus intereses económicos.

Dichas etapas no son rígidas - en el caso de la construcción-, ya que cualquier desfase temporal requiere de reprogramaciones que inciden, naturalmente, sobre los costos.

De igual manera, pueden presentarse cambios de diseño sobre la marcha que deben costearse responsablemente para evitar que los contratistas pierdan dinero o vean comprometida su reputación.

No existen tampoco patrones universales para agrupar actividades en paquetes de trabajo o para la identificación de hitos; sin embargo, en la medida que los responsables de las obras sean expertos, cuenten con experiencia previa y conozcan la naturaleza y destino de las obras, más fácil les resultará diseñar un EDT útil para el control de la ejecución de las obras.

De igual manera, al organizar razonablemente bien la logística de la construcción en función de su grado de avance, se propician reducciones de costo durante el desarrollo.
El diseño de las actividades de construcción debe ir acompañado de un costeo responsable, a fin de disponer de las referencias apropiadas para la supervisión y el control del proyecto.

Al combinar el alcance de las actividades, el programa de ejecución y los costos asociados con cada una de sus etapas, resulta práctico usar herramientas convencionales de seguimiento como las contenidas en la Guía del PMBOK elaborada por el Project Management Institute, Inc (PMI).

El control profesional del proyecto de construcción puede hacer la diferencia entre resultados financieros favorables o desfavorables para los contratistas, considerando que la improvisación puede ignorar detalles importantes para cumplir con los plazos de entrega y mantener los costos controlados.

\section{Curvas "s"}

Ahora bien, al sobreponer sobre la programación de actividades relacionadas con la ejecución de una obra (Gráfico de Gantt), el valor acumulado de los costos incurridos al realizarlas, se construyen figuras curvas que semejan una $\mathrm{S}$, de donde proviene el nombre de las referidas curvas que constituyen una verdadera herramienta de control de costos al darle seguimiento a las actividades a través de períodos regulares de tiempo, como semanas o meses; se apreciaría así cómo los costos comienzan a subir gradualmente al inicio de las obras, experimentándose un incremento significativo, con poste- 
rioridad, para luego reportarse incrementos marginales al final de la construcción.

Al graficar esta información en el primer cuadrante de un plano cartesiano donde las abscisas corresponderían al tiempo y las ordenadas, a los costos, se apreciarían figuras como las que aparecen a continuación:

Gráfico 1: Curvas "s"

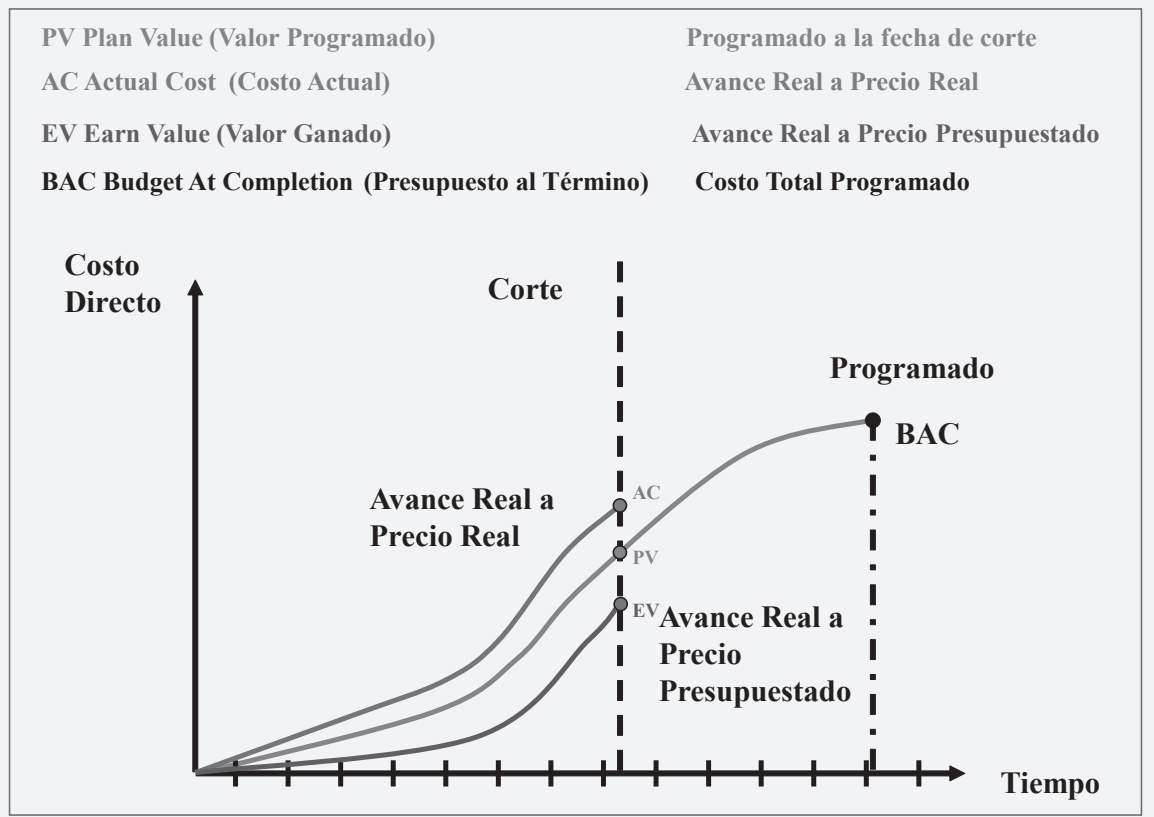

Fuente: ECOSOFT.

A partir de los paquetes de trabajo identificados para el control de los avances de la obra y su respectivo costo, se elabora un presupuesto global que se va ejecutando a lo largo del tiempo, en función de la terminación de cada uno de los paquetes de entregables programados; dicho presupuesto sirve como línea base de control, denominándosele en el argot de la gestión de proyectos: Budget At Completion BAC (presupuesto al término); dicha línea está representada aquí con un color verde. Contra esta línea base del presupuesto se verifica, cada cierto tiempo -fecha estado-, cuál es el progreso logrado por las obras físicas de la construcción, así como los costos acumulados a esa fecha. 
El avance real de la obra, según los costos presupuestados por cada paquete de trabajo se representan en este gráfico mediante la curva azul; mientras que la curva roja representa ese mismo avance físico, sólo que expresado, en esta ocasión, en términos de costos reales, no presupuestarios.

De la simple inspección de la gráfica se deduce, entonces, que la obra se encuentra retrasada (EV-PV) y que los costos se hallan excedidos, tomando en cuenta lo presupuestado para ese nivel de avance (EV-AC).

\section{Valor ganado}

Dentro del ámbito del control de los proyectos se utiliza un concepto cuyos orígenes se remonta a más de 60 años atrás, el cual fue instaurado por las Fuerzas Armadas de los EE UU, el cual se ha venido extendiendo sistemáticamente gracias a la contribución del PMI y sus guías de trabajo (PM$\mathrm{BOK})$, estando vigente, desde hace algunos años, su quinta versión donde se introducen nuevas herramientas de control de tiempos de ejecución.

Este concepto de Valor Ganado corresponde al grado de ejecución de las obras - a una fecha determinada-, versus el programa de trabajo original para la referida fecha; todo esto expresado en términos de costos estimados. A esta medida de avance se le denomina Valor Ganado, identificándosele mediante las siglas EV (Earned Value); mientras que el trabajo planificado se identifica con las siglas PV (Planned Value) ${ }^{1}$; la diferencia que surge entre estas dos categorías nos indica el grado de desviación entre el plan y la ejecución; a esta variación se le identifica normalmente con las siglas SV (Scheduled Variance), aunque algunos autores se refieren a este indicador mediante la expresión VP, utilizando las siglas en español para referirse a la Variación de la Programación.

Esta herramienta de Valor Ganado permite reducir la subjetividad en el control de los proyectos, ya que se refiere a paquetes de trabajo claramente identificados que pueden estar terminados, avanzados o totalmente retrasados - a la fecha de evaluación-, respecto a la programación; al estar completamente terminados se les imputan los costos estimados correspondientes. Al comparar las actividades planeadas versus las ejecutadas, al costo estimado, se establece una diferencia, en términos de dinero; esta diferencia resultante de restarle al EV el PV, nos indica, mediante su signo si estamos adelantados, en tiempo o rezagados; un valor negativo sugiere claramente que el proyecto está rezagado y viceversa.

De igual manera al relacionar ambas categorías EV y PV, deducimos un nuevo indicador de control que se denomina SPI (Schedule Performance Index); al dividir el EV entre el PV deducimos un índice de desempeño útil para el control del proyecto que muestra el grado de avance o demora relativa de la obra; un valor superior a 1 indicaría el porcentaje de avance por encima de lo planificado, a una fe-

1 El PV a una fecha estado se encuentra sobre la curva BAC. 
cha determinada; si este indicador es inferior a 1 , reflejaría cuál es el porcentaje de demora existente; siendo 1 la meta para esa fecha.

Este índice de desempeño sobre el avance del proyecto resulta útil hasta cierto nivel de progreso de la construcción, ya que en la medida que se acerca la finalización de la obra, dicho indicador tiende a subestimar el atraso de manera progresiva, es decir que, mientras más cerca está la finalización, menor desviación reporta el índice SPI.

Es precisamente por esa razón de subestimación, que el PMI ha ampliado sus programas de formación hacia la divulgación del concepto de Programación Ganada, como una extensión natural de la definición de Valor Ganado, aunque medido aquí en términos de tiempo, a diferencia del indicador EV expresado en dinero.

El Valor Ganado también se evalúa en términos de costos incurridos a una fecha determinada, a través del indicador AC (Actual Cost), que se refiere al costo acumulado a una fecha específica; al introducir este indicador en el análisis se puede apreciar si los costos del proyecto, al nivel de avance en que se encuentra, están dentro de márgenes de tolerancia razonables o si los costos reportados, a pesar de encontrarse por debajo de los estimados, no corresponden a la suma que debería haberse erogado, dado el avance alcanzado a la fecha.

En otras palabras, de encontrarse el AC por encima del EV estaríamos excedidos en costos; la diferencia entre el Valor Ganado y el costo real o AC, se identifica mediante las siglas CV (Cost Variance); cuando dicho indicador, expresado en términos de unidades monetarias, es negativo, podría asegurarse que los costos superan lo programado, siendo así una señal de alarma para la implementación de medidas correctivas.

$\mathrm{Al}$ reordenar esas dos categorías (EV y AC) se obtiene un indicador de desempeño de utilidad indiscutible, denominado CPI (Cost Performance Index); al referirnos a este indicador mediante el calificativo de indiscutible nos sujetamos al hecho que, a diferencia del SPI, no presenta sesgo alguno al acercarse la finalización del proyecto; siempre mostrará si los costos se excedieron de lo planificado o no.

Cabe enfatizar aquí, que los indicadores CV y CPI constituyen una herramienta útil para el control de costos, ya que el primero refleja situaciones de sobrecostos en términos absolutos, por ejemplo, cuando su valor tiene signo negativo; mientras que el segundo indicador nos brinda un dato relativo sobre la eficiencia en el uso de los recursos financieros, ya que un coeficiente inferior al 1 supondría, en cierta forma, cuánto de avance del proyecto se ha logrado por dólar de presupuesto.

La utilidad del Análisis del Valor Ganado (AVG), en términos de desviación de la programación, dado que los indicadores están expresados en dinero, no resulta tan categórica como sucede en el caso de los costos, especialmente cuando nos acercamos a la finalización del proyecto y el SV tiende a disminuir de manera progresiva, en función de la culminación de la obra. 
A continuación se presentan las siglas o acrónimos de los indicadores convencionales uti- lizados para el control de los costos y avances físicos de un proyecto de construcción:

\section{Cuadro 1: Categorías e indicadores sobre la gestión de proyectos}

\begin{tabular}{|c|c|c|}
\hline Acrónimo & Término & Interpretación \\
\hline PV & Planned Value (Valor Planeado) & $\begin{array}{l}\text { Al día de hoy cuál es el valor estimado del } \\
\text { trabajo planificado. }\end{array}$ \\
\hline EV & Earned Value (Valor Ganado) & $\begin{array}{l}\text { Al día de hoy cuál es el valor estimado del } \\
\text { trabajo realizado. }\end{array}$ \\
\hline AC & Actual Cost (Costo Actual) & $\begin{array}{l}\text { Al día de hoy cuál es el costo del trabajo } \\
\text { realizado. }\end{array}$ \\
\hline BAC & $\begin{array}{l}\text { Budget at Completion } \\
\text { (Presupuesto) }\end{array}$ & Monto presupuestado para todo el proyecto. \\
\hline EAC & $\begin{array}{l}\text { Estimate at Completion } \\
\text { (Costo Total) }\end{array}$ & ¿Cuánto costará todo el proyecto? \\
\hline ETC & Estimate to Complete & $\begin{array}{l}\text { Cuál es el costo pendiente de llevar acabo para } \\
\text { terminar el proyecto. }\end{array}$ \\
\hline VAC & Variance at Completion & $\begin{array}{l}\text { Al día de hoy cuánto esperamos que sea la } \\
\text { desviación con respecto al presupuesto. }\end{array}$ \\
\hline Cost Variance (CV) & EV-AC & $\begin{array}{l}\text { Negativo representa estar sobre el presupuesto, } \\
\text { positivo representa estar bajo el presupuesto. }\end{array}$ \\
\hline $\begin{array}{l}\text { Schedule Variance } \\
\text { (SV) }\end{array}$ & EV-PV & $\begin{array}{l}\text { Negativo es atrasado en tiempo, positivo es } \\
\text { adelantado en el tiempo. }\end{array}$ \\
\hline $\begin{array}{l}\text { Cost Performance } \\
\text { Index (CPI) }\end{array}$ & $\mathrm{EV} / \mathrm{AC}$ & Estamos obteniendo \$X de cada \$1 invertido. \\
\hline $\begin{array}{l}\text { Estimate at } \\
\text { Completion (EAC) }\end{array}$ & $\begin{array}{l}E A C=B A C / C P I \\
E A C=A C+E T C \\
E A C=A C+(B A C-E V)\end{array}$ & $\begin{array}{l}\text { Al día de hoy, cuánto estimamos el proyecto to- } \\
\text { tal que cueste. }\end{array}$ \\
\hline $\begin{array}{l}\text { To complete } \\
\text { performance Index }\end{array}$ & $\begin{array}{l}\mathrm{TCPI}=(\mathrm{BAC}-\mathrm{EV}) /(\mathrm{BAC}-\mathrm{AC}) \\
\mathrm{TCPI}=(\mathrm{BAC}-\mathrm{EV}) /(\mathrm{EAC}-\mathrm{AC})\end{array}$ & $\begin{array}{l}\text { Para mantenernos dentro del presupuesto cuál } \\
\text { debe ser nuestro índice de desempeño. }\end{array}$ \\
\hline $\begin{array}{l}\text { Estimate to } \\
\text { complete }\end{array}$ & $\begin{array}{l}\mathrm{ETC}=\mathrm{EAC}-\mathrm{AC} \\
\mathrm{ETC}=\mathrm{BAC}-\mathrm{EV}\end{array}$ & ¿Cuánto más costará el proyecto? \\
\hline $\begin{array}{l}\text { Variance at } \\
\text { completion }\end{array}$ & $V A C=B A C-E A C$ & ¿Cuál será la desviación al final del proyecto? \\
\hline
\end{tabular}

Fuente: Project Managment Institute. 
Ahora bien, el propósito del control consiste en corregir desviaciones significativas entre la fecha prevista de entrega de la obra y el grado de avance identificado a una fecha de corte particular -fecha estado-; es precisamente para esto que se dispone de los índices de desempeño SPI y CPI, ya que está demostrado, estadísticamente hablando, que el $70 \%$ de los proyectos registran sobrecostos y se atrasan y que, además, el 52\% de los proyectos registran costos cercanos al $190 \%$ de su presupuesto original; mientras que algunos de ellos simplemente no se logran terminar.

Por lo tanto, el "Análisis del Valor Ganado" sirve para estimar el costo que supondría culminar los proyectos a partir de una fecha determinada.

La diferencia entre el presupuesto inicial (BAC) y el presupuesto estimado al finalizar el proyecto (EAC) permitiría contar con un dato aproximado acerca de la desviación entre el valor estimado original y el costo estimado al vencimiento, a partir de una fecha específica, claro está.

Cabe señalar, en todo caso, que el valor estimado al vencimiento puede calcularse tomando en cuenta diversos supuestos; en función de ellos, el costo estimado al vencimiento diferiría entre un método y otro.

Si se supone, por ejemplo, que el índice de desempeño en costos (CPI) refleja una situación que se mantendría a lo largo del proyecto, el costo total al final del proyecto se establecería al dividir el BAC entre el CPI.
Esto supondría que las variaciones observadas en materia de costos a la fecha estado se mantendrían a lo largo del proyecto y, por lo tanto, afectarían sistemáticamente los costos de ejecución.

Naturalmente que antes de estimar mecánicamente el EAC a partir del CPI, sería necesario indagar sobre las causas que explican una inadecuada gestión de costos, de ser el caso, ya que al identificarlas pueden adoptarse medidas para mitigar su impacto sobre los costos totales a la finalización del proyecto.

En esa misma línea de supuestos, podríamos considerar que, si el índice de desempeño del cronograma (SPI) reproduce una situación que continuará incidiendo sobre la ejecución del proyecto, a futuro, se conjugaría el sobrecosto con el atraso en la ejecución de la obra y para calcular el EAC, lo más adecuado sería dividir el BAC entre el producto del CPI y el SPI. En este caso consideraríamos que la variación en costos y cronograma, son típicas del proyecto, ergo, afectarían los costos de manera permanente.

En todo caso, deberían explorarse alternativas para corregir los excesos de gastos mediante outsourcing, la reprogramación del trabajo para reducir tiempos ociosos y el pago de horas extras, la contratación de mano de obra menos calificada en tareas que no sean claves para cumplir con las especificaciones de la construcción, el arrendamiento de equipo, etc.

Habría que agregar aquí, que al producto entre el CPI y el SPI se le conoce con el 
nombre de Índice de Costo-Programación (CSI), sabiéndose que mientras más se aleje dicho índice de 1, menos probabilidades ha- brá de que el proyecto se recupere, según lo señalan expertos en el tema.

\section{Cuadro 2: índice de costo - programación}

\begin{tabular}{cc}
\hline Índice & Nivel de Alarma \\
$0.9<\mathrm{CSI}<1.2$ & OK \\
$0.8<\mathrm{CSI}<0.9 \circ 1.2<\mathrm{CSI}<1.3$ & CHEQUEE \\
$\mathrm{CSI}<0.8 \circ \mathrm{CSI}>1.3$ & BANDERA ROJA \\
\hline
\end{tabular}

Fuente: BG Bernardo García, Adiestramiento y Asesoría.

En el cuadro 2 se identifican rangos de control según la lógica de los colores del semáforo; el primer rango sería un verde, el siguiente correspondería a amarillo; mientras que el último sería rojo que debería disparar las alarmas de los responsables de la gestión del proyecto.
A continuación se presentan de forma esquemática las fórmulas y el significado de los principales indicadores de control de proyectos:

\section{Gráfico 2: fórmulas de indicadores de gestión de proyectos}

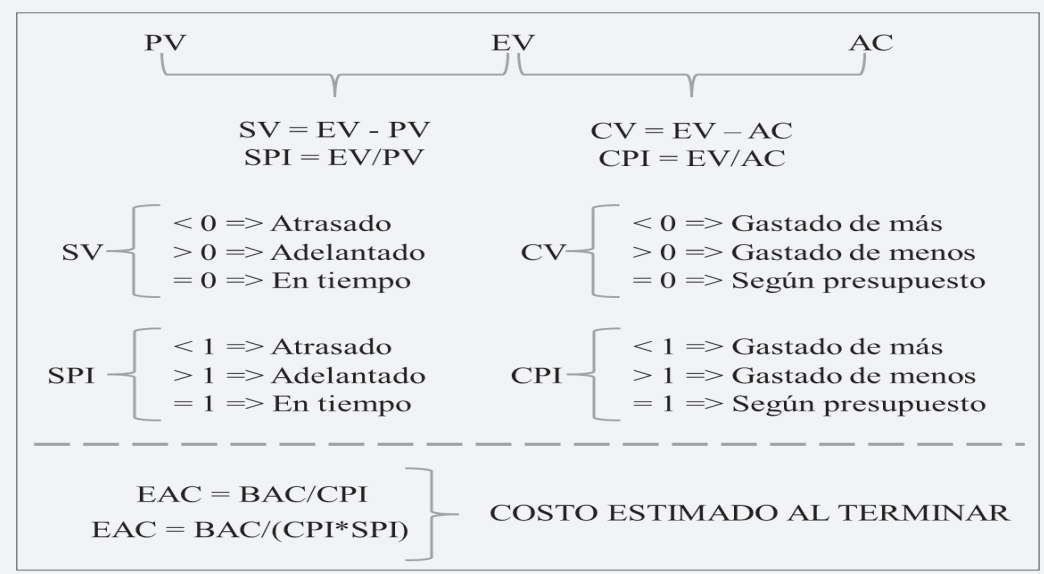

Fuente: Elaboración propia. 
En la parte superior se observa que los valores de donde se originan los indicadores de desviación y de desempeño son el PV, EV y AC, con los cuales podemos medir el grado de cumplimiento del programa y de los costos presupuestados por entregable; mientras que los índices reflejan, de alguna manera, la eficiencia en el uso de los recursos financieros y del tiempo previsto para la conclusión de las obras.

De igual manera, en la parte inferior del Gráfico 2 se destaca la utilidad del AVG para proyectar los costos que supondría la terminación de la obra, considerando la tipicidad en la eficiencia de los costos y de la programación, medida a través del CSI.

Para finalizar este apartado y a manera de conclusión sobre la importancia del control de proyectos, en general y de sus costos, en particular, me gustaría citar a Norberto Figueroa quien sostiene que: "Un proyecto llevado a cabo sin la gestión de control de costo y técnicas de proyecciones de presupuesto, probablemente estará en lista para un posible sobre-costo. Una supervisión del presupuesto hecha frecuentemente evita estos problemas. Un presupuesto controlado con tiempo con un 10 por ciento de desvío es mucho más fácil de corregir que uno descubierto más tarde con un 50 por ciento. Las posibilidades de mantener el proyecto en línea a través de las frecuentes revisiones del costo es mucho mayor que si hacemos el presupuesto y nos olvidamos de él". De ahí que, la gestión profesional de proyectos resulte crítica para cumplir con los tiempos de entrega y el control de los costos en la industria de la construcción, es decir, para el éxito financiero de los inversionistas que desarrollan esta actividad.

\section{Gestión de costos}

Una vez se cuenta con una línea base de control y monitoreo sobre la ejecución de proyectos se verifican periódicamente las desviaciones registradas, tanto en materia de cronograma como de presupuesto.

Ahora bien, dicho monitoreo tiene como propósito corregir, oportunamente, desviaciones peligrosas que dificulten la entrega oportuna de las obras o que revelen incrementos sustanciales de costos, impidiendo con ello que los contratistas obtengan utilidades razonables.

Esto significa que en caso de observarse incrementos importantes, respecto a lo presupuestado, en función de los hitos alcanzados, será necesario evaluar los entregables con los contratantes, a efecto de identificar trabajos que puedan modificarse o suprimirse por completo, para mantener la ejecución dentro del programa original.

En cuanto a los costos se refiere, será necesario examinar la calificación requerida del recurso humano y las holguras entre una actividad y otra, a efecto de sustituir trabajo calificado por uno de menor costo cuando el reemplazo no represente riesgos importantes para la entrega del proyecto o de incumplimiento de requisitos técnicos, esto por un lado; mientras que la gestión precisa del recurso humano puede reducir 
los tiempos muertos y optimizar el uso de presupuesto, por el otro.

Como ya se mencionó anteriormente, la proyección del costo al término de las obras nos permite establecer, además, el nivel de eficiencia que debe lograrse en lo que resta del proyecto para alcanzar los objetivos trazados.

Para esto se utiliza el Índice de Desempeño Para Completar (TCPI). Este indicador relaciona el trabajo pendiente con el realmente ejecutado; en tal sentido, si este indicador es mayor a 1 representaría que los trabajos deberían acelerarse por encima de la planificación original para controlar los costos. Todo esto expresado en términos de unidades monetarias.

Se puede deducir entonces que el índice TCPI resultaría de dividir el presupuesto no ejecutado (BAC-EV) entre el ejecutado (BAC-AC). En otras palabras, medimos el trabajo pendiente, respecto al presupuesto pendiente, expresados ambas diferencias en unidades monetarias para convertirlas en un valor relativo.

$$
T C P I=\frac{(B A C-E V)}{(B A C-A C)}
$$

Si este indicador resulta positivo, habrá que redoblar el esfuerzo para cerrar la brecha respecto al programa original.

Sin embargo, si como consecuencia del AVG se ajusta el presupuesto en función del ETC, el indicador cambiaría en el siguiente sentido:

$$
T C P I=\frac{(B A C-E V)}{(E A C-A C)}
$$

Normalmente, al haberse aprobado los cambios en los parámetros de costos, la presión se relaja un puede trabajarse más tranquilamente, aunque no deberíamos dejar de controlar la ejecución de la obra con el mismo nivel de responsabilidad, a fin de evitar que los costos se vuelvan a salir del rango previsto, manteniendo informado al equipo encargado de la obra para mejorar la autogestión.

Para efectos de ejemplo, asumamos que un proyecto ha sido descompuesto en 4 entregables (paquetes de trabajo) y que se cuenta con 4 semanas para terminarlo, siendo el costo estimado de $\$ 6,000$, tal como aparece a continuación. 
Cuadro 3: Estructura de desglose de trabajo EDT de un proyecto " $x$ "

\begin{tabular}{lrrrrrr}
\hline \multicolumn{1}{c}{ EDT } & Semana 0 & Semana 1 & Semana 2 & Semana 3 & Semana 4 & Total \\
\hline Tarea 1 & $\$ 100$ & & & $\$ 100$ \\
Tarea 2 & $\$ 500$ & $\$ 500$ & & & $\$ 1,000$ \\
\hline Tarea & & $\$ 1,000$ & $\$ 1,500$ & & $\$ 2,500$ \\
Tarea 4 & & & $\$ 1,500$ & $\$ 900$ & $\$ 2,400$ \\
Proyecto & $\$ 0$ & $\$ 600$ & $\$ 1,500$ & $\$ 3,000$ & $\$ 900$ & $\$ 6,000$ \\
Acumulado & $\$ 0$ & $\$ 600$ & $\$ 2,100$ & $\$ 5,100$ & $\$ 6,000$ & \\
\hline
\end{tabular}

Fuente: Elaboración propia.

Al final de la tercera semana se revisa el avance de cada tarea, hallándose la siguiente situación:

Cuadro 4: Control de avance

\begin{tabular}{lcccccc}
\hline EDT & Semana 0 & Semana 1 & Semana 2 & Semana 3 & Semana 4 & Total \\
\hline Tarea 1 & $20 \%$ & $80 \%$ & & & $100 \%$ \\
\hline Tarea 2 & $40 \%$ & $40 \%$ & $20 \%$ & $100 \%$ \\
\hline Tarea & & $40 \%$ & $50 \%$ & $90 \%$ \\
\hline Tarea 4 & & & $50 \%$ & $50 \%$ \\
\hline
\end{tabular}

Fuente: Elaboración propia.

De los cuatro paquetes de trabajo, dos ya han concluido, el tercero tiene un 90\% de avance; mientras que el cuarto solamente un 50\%.
Según los costos previstos por actividad, el Valor Ganado sería el siguiente: 


\section{Cuadro 5: Valor ganado en EV}

\begin{tabular}{lrrrrrr}
\hline \multicolumn{1}{c}{ EDT } & Semana 0 & Semana 1 & Semana 2 & Semana 3 & Semana 4 & Total \\
\hline Tarea 1 & $\$ 20$ & $\$ 80$ & & & $\$ 100$ \\
\hline Tarea 2 & & $\$ 400$ & $\$ 400$ & $\$ 200$ & $\$ 1,000$ \\
\hline Tarea & & $\$ 1,000$ & $\$ 1,250$ & $\$ 2,250$ \\
\hline Tarea 4 & & & $\$ 1,250$ & $\$ 1,200$ \\
\hline Proyecto & $\$ 20$ & $\$ 480$ & $\$ 1,400$ & $\$ 2,650$ & $\$ 4,550$ \\
\hline Acumulado & $\$ 20$ & $\$ 500$ & $\$ 1,900$ & $\$ 4,550$ & \\
\hline
\end{tabular}

Fuente: Elaboración propia.

En el cuadro anterior observamos, por ejemplo, que antes del inicio de la ejecución planificada del proyecto, ya se contaba con un $20 \%$ de avance en la tarea 1 , registrándose entonces un $\$ 20(20 \%$ de la tarea 1 cuyo presupuesto era de $\$ 100$ ) para la semana 0 .

En la semana 1 se completó dicha tarea, por lo que aparece en el cuadro $\$ 80$, en esa línea; sumándose así los $\$ 100$ presupuestados para la tarea; pero además, se tenía un avan- ce del $40 \%$ de la tarea 2 , equivalente a $\$ 400$ (40\% de la tarea 2 cuyo presupuesto era de $\$ 1,000)$; en esa semana se obtuvo un EV de \$480, acumulándose, por ende, $\$ 500$ de EV a esa "fecha estado".

Sin embargo, lo efectivamente gastado en las actividades realizadas durante la primera semana fueron $\$ 430$, acumulándose, por lo tanto, un AC de $\$ 450$ para dicha fecha, tal como se ilustra a continuación:

\section{Cuadro 6: Costo real AC}

\begin{tabular}{lrrrrrr}
\hline \multicolumn{1}{c}{ EDT } & Semana 0 & Semana 1 & Semana 2 & Semana 3 & Semana 4 & Total \\
\hline Tarea 1 & $\$ 20$ & $\$ 80$ & & & $\$ 100$ \\
\hline Tarea 2 & & $\$ 350$ & $\$ 400$ & $\$ 150$ & $\$ 900$ \\
\hline Tarea & & $\$ 950$ & $\$ 1,000$ & $\$ 1,950$ \\
\hline Tarea 4 & & & $\$ 1,300$ & $\$ 1,300$ \\
\hline Proyecto & $\$ 20$ & $\$ 40$ & $\$ 1,350$ & $\$ 2,450$ & $\$ 4,250$ \\
\hline Acumulado & $\$ 20$ & $\$ 40$ & $\$ 1,800$ & $\$ 4,250$ & \\
\hline
\end{tabular}

Fuente: Elaboración propia. 
De la estructura de desglose original se deduce la línea base para el seguimiento del proyecto; de ahí que, para la semana aquí analizada, la planificación de avances suponía tener completa la tarea 1 y la tarea, ter- minada en un $50 \%$; previéndose un gasto planificado de $\$ 600$.

Bajo la lógica anterior, al ubicarnos en la tercera semana, tendríamos el siguiente cuadro resumen:

\section{Cuadro 7: Resumen}

\begin{tabular}{lccccc}
\hline & Semana 0 & Semana 1 & Semana 2 & Semana 3 & Semana 4 \\
Presupuesto & $\$ 0$ & $\$ 600$ & $\$ 2,100$ & $\$ 5,100$ & $\$ 6,000$ \\
\hline Costo Real & $\$ 20$ & $\$ 450$ & $\$ 1,800$ & $\$ 4,250$ & \\
Valor ganado & $\$ 20$ & $\$ 500$ & $\$ 1,900$ & $\$ 4,550$ & \\
\hline
\end{tabular}

Fuente: Elaboración propia.

Al graficar estos valores en formato tipo "S", se tendrían las siguientes figuras:

\section{Gráfico 3: BAC, VP, EV, AC del proyecto}

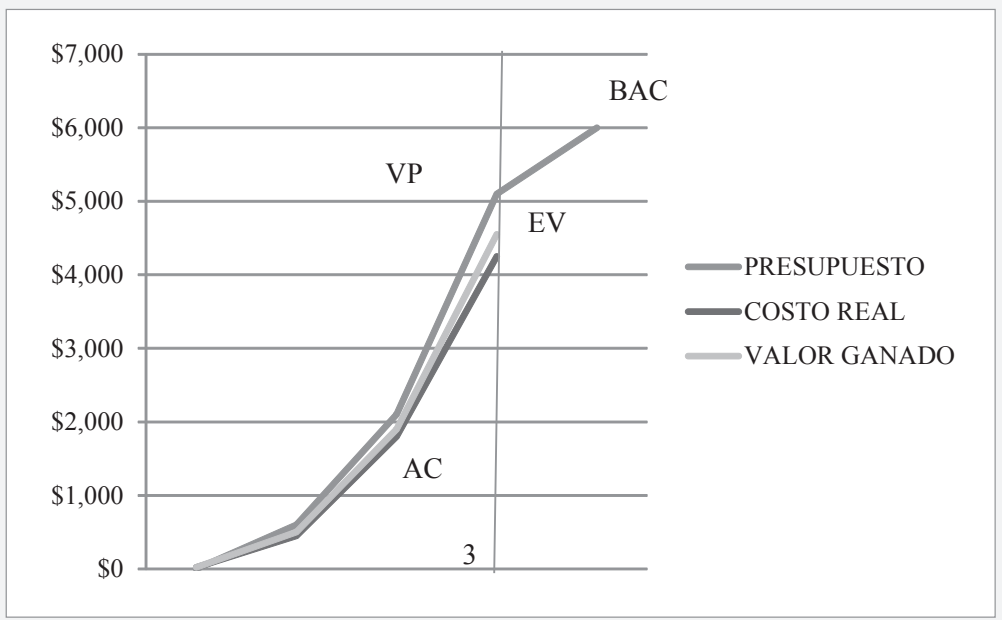

Fuente: Elaboración propia.

Se observa claramente que el EV está por debajo del VP, hallándose el proyecto reza- go respecto al cronograma; sin embargo, el EV está por encima del AC, lo cual indica 
que existe una desviación favorable respecto a los costos, es decir, se ha gastado menos de lo programado para el nivel de avance logra- do efectivamente. Esta apreciación visual se confirma al construir indicadores de gestión, tal como aparece en el siguiente cuadro.

\section{Cuadro 8: Indicadores de gestión de proyectos}

\begin{tabular}{cc}
\hline Varianza de costos & Indices de desempeño \\
$\mathrm{CV}=\mathrm{EV}-\mathrm{AC}$ & 1.07 \\
$\$ 300$ & \\
Varianza del cronograma & $\mathrm{AC}$ \\
$\mathrm{SV}=\mathrm{EV}-\mathrm{PV}$ & $\mathrm{SPI}=\mathrm{EV} / \mathrm{PV}$ \\
$-\$ 550$ & 0.89 \\
\hline
\end{tabular}

Fuente: Project Managment Institute.

Queda claro, a partir del cuadro anterior, que el proyecto en términos de costo está bien $\mathrm{CPI}=1.07$; pero rezago, $\mathrm{SPI}=0.89$; para un CSI de 0.96. De mantenerse estos indica- dores de gestión en costos y programación, se podría espera que los costos al terminar el proyecto excedieran los $\$ 6,000$, tal como aparece en la siguiente gráfica.

\section{Gráfico 4: EAC, BAC, VP, EV, AC del proyecto}

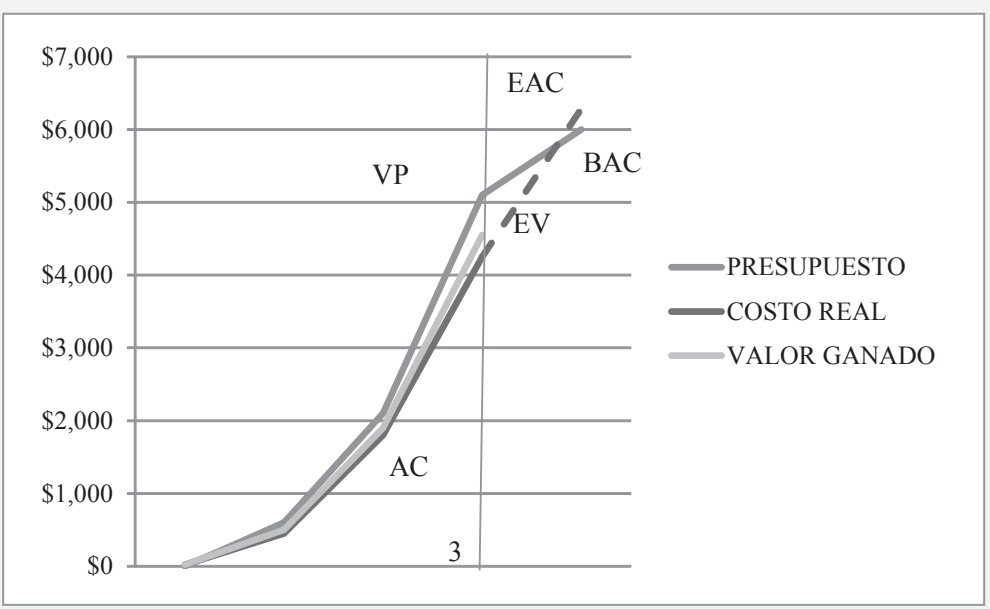

Fuente: Elaboración propia. 
La línea roja punteada correspondería a la estimación de los costos al término del pro- yecto (EAC), excediendo el valor del BAC, tal como se estima en el siguiente cuadro.

\section{Cuadro 9: Indicadores de gestión de proyectos}

\begin{tabular}{c}
\hline Indice Costo-Programación \\
$\mathrm{CSI}=\mathrm{CP}{ }^{*} \mathrm{SPI}$ \\
0.96 \\
Estimación a la terminación \\
$\mathrm{EAC}=\mathrm{BAC} / \mathrm{CPI}$ \\
$\$ 6,282$ \\
\hline
\end{tabular}

Fuente: Project Managment Institute.

De autorizarse las órdenes de cambio correspondientes y ajustarse el presupuesto a \$6,282, el Índice de Desempeño para Completar (TCPI) rondaría 0.71 , lo cual resulta bastante confortable; sin embargo, en caso de no aprobarse la orden de cambio del pre- supuesto, siempre se contaría con holgara suficiente para no tener que correr con los tiempos, ya que con los datos originales, el TCPI ascendería 0.83, aproximadamente, como se muestra continuación.

\section{Cuadro 10: Indicadores de gestión de proyectos}

\begin{tabular}{cc}
\hline \multicolumn{2}{c}{ Con Presupuesto } \\
\hline BAC-EV & $\$ 1,450$ \\
\hline BAC-AC & $\$ 1,750$ \\
\hline TCPI & 0.83 \\
\hline
\end{tabular}

\begin{tabular}{cc}
\hline \multicolumn{2}{c}{ Con Ajuste de Presupuesto } \\
\hline BAC-EV & $\$ 1,450$ \\
\hline EAC-AC & $\$ 2,032$ \\
\hline TCPI & 0.71 \\
\hline
\end{tabular}

Fuente: Project Managment Institute.

Según los indicadores de gestión, el proyecto se estaría ejecutando dentro de los parámetros previstos y no se tendría mayor presión para acelerar el trabajo, ni para adoptar medidas correctivas significativas para el control de costos.

\section{Conclusiones}

La gestión de proyectos de construcción es clave para su éxito financiero, tanto como la planificación y su evaluación económica, ya que las desviaciones de programa y costos 
pueden ocasionar la ruina de los contratistas y el abandono de las obras, en algunas oportunidades.

El Project Managment Institute ha sistematizado a través de sus guías, pautas, metodologías e indicadores de control que permiten identificar oportunamente las desviaciones registradas durante la ejecución de obras de construcción.

El Análisis del Valor Ganado constituye una herramienta fundamental en ese sentido, contándose indicadores de alerta para que los responsables de la gestión implementen medidas correctivas que permitan mantenerse dentro de programa y presupuesto.

El Indicador de Desempeño para Completar el proyecto nos brinda una idea de la velocidad con que deben realizarse las obras pendientes para evitar desviaciones peligrosas, aun cuando se haya logrado ajustar el presupuesto mediante órdenes de cambio.

\section{Referencias bibliográficas}

1. Navarro, Diego; Seguimiento de proyectos con el Análisis del Valor Ganado; http://direccion-proyectos. blogspot.com/dnavarro@armell.com

2. Project Managment Institute; Gestión del Valor Ganado del Trabajo en Curso, 2010.

3. Project Managment Institute; Guía de los FUNDAMENTOS PARA LA DIRECCIÓN DE PROYECTOS (GUIA DEL PMBOK), Quinta Edición.

4. Sorto Rivas, Francisco; apuntes de clases de la cátedra COSTEO DE ACTIVIDADES DEL PROYECTO, Universidad Doctor José Matías Delgado, 2016.

5. Sorto Rivas, Francisco; apuntes de clases de la cátedra Evaluación de Proyectos, Instituto Superior de Economía y Administración de Empresas ISEADE, 2016.

6. Sorto Rivas, Francisco; Gestión de proyectos de construcción, Revista Empresa, Instituto Superior de Economía y Administración de Empresas ISEADE, 2016. 